\title{
Scanning Ellipsometer Using a Fixed Phase Retarder and Rotating Polarizer and Analyzer
}

\author{
S.A. TAYA* AND T.M. EL-AgEZ \\ Physics Department, Islamic University of Gaza, P.O. Box 108, Gaza, Palestine
}

\begin{abstract}
A spectroscopic rotating polarizer-analyzer ellipsometer in which the polarizer and the analyzer rotate in opposite directions at the same speed and with a fixed retarder is proposed and investigated theoretically. The fixed phase retarder is introduced after the rotating polarizer to significantly reduce the percent error in the optical parameters.
\end{abstract}

DOI: 10.12693/APhysPolA.123.183

PACS: 07.60.Fs, 02.30.Nw

\section{Introduction}

Ellipsometry have been widely used in thin film characterization. It has proven to be an extremely powerful tool to study the optical properties of materials with high optical data precision in measurement. Continuous efforts are conducted to develop different theoretical models and experimental ellipsometric techniques. Among the various configurations of spectroscopic ellipsometers (RPAEs), the model often used is the rotating analyzer ellipsometer [1]. Rotating polarizer-analyzer ellipsometers in which the polarizer and the analyzer rotate with different speed ratios were proposed and constructed [2-6]. In this work, a spectroscopic RPAE with a fixed retarder with retardance $\delta$ is proposed. Hypothetical noise is assumed to be imposed on the signal received by the detector. The effect of the phase retarder in reducing the percent error in the optical constants extracted from the ellipsometric parameters is shown.

\section{Theory}

An ellipsometer measures two angles $\psi$ and $\Delta: \psi$ is the angle whose tangent is the ratio of the magnitudes of the reflection coefficients and $\Delta$ is the change in the phase differences that occurs upon reflection, i.e. $\tan \psi=\left|r_{p}\right| /\left|r_{s}\right|$, and $\Delta=\delta_{p}-\delta_{s}$, where $r_{p}$ and $r_{s}$ are the Fresnel reflection coefficients and $\delta_{p}$ and $\delta_{s}$ are the phase shifts upon reflection for $E_{p}$ and $E_{s}$ components. The fundamental ellipsometric equation is conventionally written as $\rho=r_{p} / r_{s}=\tan (\psi) \exp (\mathrm{i} \Delta)$.

The proposed RPAE comprises a light source, a fixed polarizer with an azimuth angle $\theta$, a polarizer rotating at an angular speed $\omega$, a fixed phase retarder, a sample, an analyzer rotating at $\omega$, and a detector. The two rotating elements are assumed to rotate in opposite directions. The fast axis of the retarder makes an angle with respect to the plane of incidence. If $\varepsilon$ and $\sigma$ are the azimuth angles of the rotating polarizer and the rotating analyzer at $t=0$, respectively, the azimuth angles of the rotating polarizer $(P)$ and the rotating analyzer $(A)$ at any time are given by $P=\omega t+\varepsilon$ and $A=-\omega t+\sigma$.

*corresponding author; e-mail: staya@iugaza.edu.ps
Theory of the Fourier ellipsometry is usually formulated in terms of Jones-matrix formalism. The transformation of polarization states by optical elements can be described by the $2 \times 2$ Jones matrix. The matrix for the fixed polarizer, rotating polarizer, fixed phase retarder, sample, and rotating analyzer can be found in Ref. [7]. The intensity received by the detector can be obtained in terms of these matrices which has the Fourier transform $I(t)=a_{0}+\sum_{n=1}^{3} a_{n} \cos 2 n \omega t+\sum_{n=1}^{3} b_{n} \sin 2 n \omega t$, where $a_{0}, a_{n}$, and $b_{n}$ are the Fourier coefficients. When the azimuth angles $\theta, \varepsilon, \sigma$, and $\beta$ are set to zero, the coefficients $b_{n}$ vanish. The nonzero Fourier coefficients $\left(a_{0}, a_{1}, a_{2}, a_{3}\right)$ can be written in terms of $\left|r_{p}\right|,\left|r_{s}\right|, \delta$, and $\Delta$ from which the ellipsometric parameters can be obtained. Three Fourier coefficients are needed to write $\psi$ and $\Delta$. Excluding the dc term $\left(a_{0}\right)$, we have three coefficients from which the ellipsometric parameters $\psi$ and $\Delta$ can be determined as

$$
\begin{aligned}
& \tan \psi=\frac{\sqrt{a_{1}+a_{3}}}{\sqrt{a_{1}-4 a_{2}+9 a_{3}}}, \\
& \cos (\Delta-\delta)=\frac{a_{1}-2 a_{2}-3 a_{3}}{\sqrt{\left(a_{1}+a_{3}\right)\left(a_{1}-4 a_{2}+9 a_{3}\right)}} .
\end{aligned}
$$

\section{Numerical results}

In our calculations, we assume an incidence angle $\theta_{0}=70^{\circ}$. One interface separating a semi-infinite air layer as an ambient and a bulk c-Si material is considered. A zero order retarder exhibits the dispersion of $\mathrm{MgF}_{2}$, where $90^{\circ}$ retardance is assumed. The Fresnel complex reflection coefficients $r_{p}$ and $r_{s}$ for $p$ and $s$ light components of such a structure are calculated. Simulated light signals are generated based on the intensity obtained in terms of the Jones matrices. The Fourier transform of the generated signal was taken to extract the coefficients $a_{0}$ through $a_{3}$. Equations (1) are then used to calculate $\psi$ and $\Delta$ in the photon energy range $1.5-4.8 \mathrm{eV}$. These values of the ellipsometric parameters correspond to the clean signal without considering any noise. In practical situations, random fluctuations in the recorded signal appear due to the noise from many sources. In order to simulate practical situations, noise was generated using MathCAD code and was superimposed on the clean signal according to the following equation: 


$$
\begin{aligned}
& I_{\text {noise }}=(\operatorname{rnd}(c)-c / 2) I+(\operatorname{rnd}(e)-e / 2) \\
& \quad+0.0001 I_{\max },
\end{aligned}
$$

where MathCAD's rnd $(c)$ function generates noise in the range from 0 to $c$ and $\operatorname{rnd}(e)$ function generates noise in the range from 0 to $e$. This noise is added to the pure signal. The Fourier transform of the noisy signal is taken to extract the new coefficients $a_{0}$ through $a_{3}$ in the presence of the noise. Equations (1) are used again to calculate the ellipsometric parameters $\psi$ and $\Delta$ for the noisy signal in the same photon energy range. The refractive index $(n)$ and the extinction factor $(k)$ of $c-S i$ are now calculated from the noisy $\psi$ and $\Delta$ parameters.
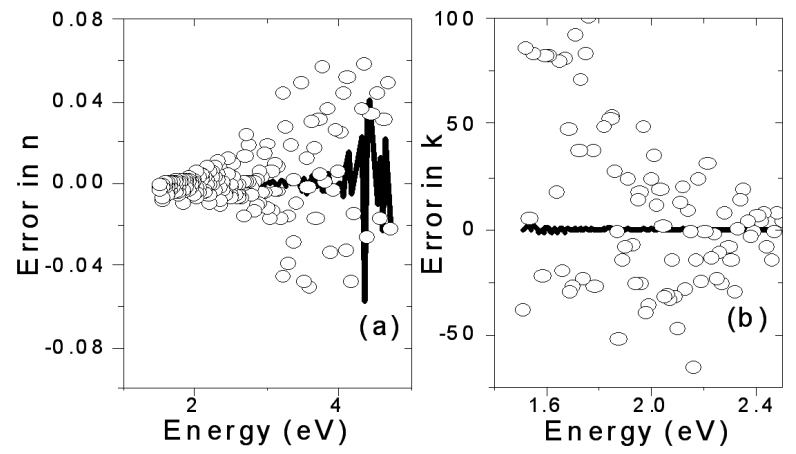

Fig. 1. Percent error in (a) the refractive index and (b) the extinction factor of c-Si as a function of energy for the ellipsometric structure with (lines) and without a retarder (points).
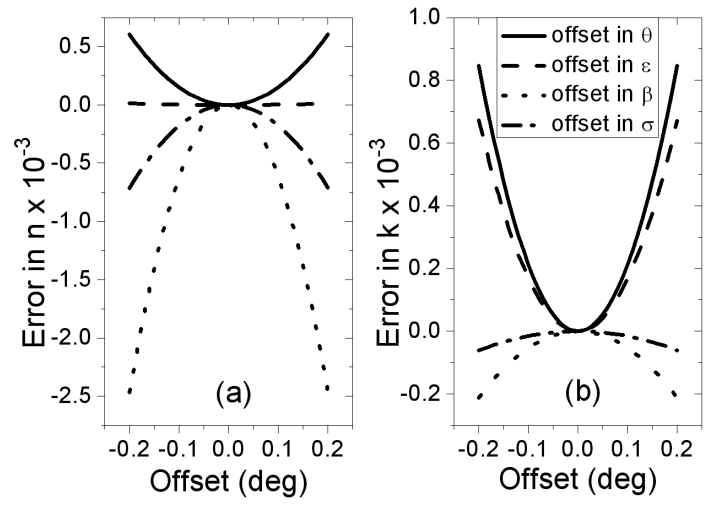

Fig. 2. Percent error in (a) $n$, (b) $k$ for c-Si sample at $\lambda=632.8 \mathrm{~nm}$ as a function of the error in $\theta, \varepsilon, \beta$, and $\sigma$ successively varied from $-0.2^{\circ}$ to $0.2^{\circ}$ while keeping the other variables equal to zero.

It is important to compare between the noise effect on the results extracted from the proposed structure without a fixed retarder and with a fixed retarder. The percent error in $n$ and $k$ of c-Si as a function of energy in the photon energy range 1.5 to $4.8 \mathrm{eV}$ for the ellipsometric structure with and without a retarder are shown in Fig. 1. Generally, the percent error in $n$ is very small in the pres- ence and absence of the retarder with a preference to the structure comprising the retarder. The preference of the structure including the retarder can be seen obviously in Fig. 2b. Due to the small value of the extinction factor $k$ in the low energy regime $(1.5-2.5 \mathrm{eV})$, the percent error in it is very high for the structure without the retarder. It approaches $\approx 270 \%$ at some points. This error can be drastically reduced with the introduction of a retarder between the rotating polarizer and the sample. The percent error ranges between $\pm 2 \%$ for the structure containing the retarder which is ignorable compared with that of the other configuration.

In practical situations, alignment of the optical elements with respect to the plane of incidence is not easy. Azimuthal misalignment of optical elements is one of the usual sources of systematic errors. Thus, it is important for the present work to study the effect of misalignment of these optical elements. Figure 2a shows the percent error in $n$ as a function of the misalignment of the fixed polarizer azimuth angle $\theta$, the rotating polarizer azimuth angle $\varepsilon$, the rotating analyzer azimuth angle $\sigma$, and the fixed retarder azimuth angle $\beta$ successively varied from $-0.2^{\circ}$ to $0.2^{\circ}$ in steps of $0.01^{\circ}$ while keeping the three other variables equal to zero. As can be seen from the figure, the impact of these errors on $n$ is not significant for small misalignment. The figure also reveals that the error due to misalignment of the azimuth angle $\varepsilon$ has almost the lowest impact. Figure $2 \mathrm{~b}$ is exactly similar to Fig. 2a but it investigates the percent error in $k$. It also reveals that the error in $k$ due to the error in alignment of all optical components is not significant.

\section{Conclusion}

Rotating polarizer-analyzer ellipsometer with a speed ratio 1:-1 and a fixed retarder has been proposed and investigated. It was applied to bulk c-Si sample. The percent error in the calculated optical parameters of c-Si has been obtained for the proposed structure with and without a fixed retarder. It was clearly shown that this error is considerably reduced in the presence of the fixed retarder. Moreover, it was shown that small misalignment of optical elements does not have a great impact on the results.

\section{References}

[1] D.E. Aspnes, Opt. Commun. 8, $222(1973)$

[2] L.Y. Chen, D.W. Lynch, Appl. Opt. 26, 5221 (1987).

[3] T.M. El-Agez, A.A. El Tayyan, S.A. Taya, Thin Solid Films 518, 5610 (2010)

[4] T.M. El-Agez, S.A. Taya, Phys. Scr. 83, 025701 (2011)

[5] T.M. El-Agez, S.A. Taya, A. El Tayayn, Int. J. Optomechatron. 5, 51 (2011).

[6] T.M. El-Agez, D.M. Wieliczka, C. Moffitt, S.A. Taya, Phys. Scr. 84, 045302 (2011)

[7] H. Fujiwara, Spectroscopic Ellipsometry Principles and Applications, Wiley, West Sussex, 2007. 